\title{
Numerical modelling of combined heat and mass transfer in film absorption
}

\author{
Maria V. Bartashevich ${ }^{1,}{ }^{*}$, Maxim G. Vlasenko ${ }^{1}$, Nadegda A. Zyuzina ${ }^{1}$, Andrey A. Pil'nik ${ }^{1}$, \\ and Andrey A. Chernov ${ }^{1}$ \\ ${ }^{1}$ Kutateladze Institute of Thermophysics, 630090 Novosibirsk, Russia
}

\begin{abstract}
Heat and mass transfer during absorption on a film of lithium bromide water solution flowing by a cooled wall in the steam atmosphere is numerically investigated in this paper. The self-similar solutions are using as the initial conditions for solving the problem beyond the entrance region. The key criteria characterizing heat and mass transfer in the film absorption with uniform velocity profile and with a constant thickness have been determined.
\end{abstract}

\section{Problem statement}

Absorption of gases or vapors by liquid takes place in various energy devices [1]. The first theoretical description of combined heat and mass transfer processes at vapor absorption in a laminar falling liquid film was made in [2-4]. The overview of works on film flows with absorption is presented in [5]. Heat and mass transfer in the processes of absorption, desorption, evaporation and condensation in the initial part of semi-infinite axisymmetric film falling under the pressure is studied in [6]. The absorption and desorption processes under the conditions, corresponding to the operating modes of thermal transformers are described in [7]. The analytical solution to the problem of conjugate heat and mass transfer in a laminar falling liquid film with a linear velocity profile is presented in [8]. Data of numerical simulation of absorption are compared with experimental data in [9].

Let us consider the two-dimensional stationary flow of a laminar liquid film (water solution of lithium bromide with constant thickness $h$ ) over a plate, inclined at angle $\theta$ to the horizon, as in [4]. The liquid surface contacts with stationary steam. Absorption is considered in the framework of usual assumptions [1]. The vapor phase is singlecomponent, and vapor pressure does not change during absorption. Thermal-physical properties of solution are considered constant. We believe that absorption heat is released at the interface and it is spent only for solution heating. For small ranges of concentrations and temperatures, according to [1], we will approximate the dependence of absorbed substance concentration on temperature by linear function $C_{i}=k_{1}-k_{2} T_{i}$, where coefficients $k_{1}$ and $k_{2}$ are determined by the pressure. The values of $C_{i}(x), T_{i}(x)$ are interrelated, unknown and should be determined.

\footnotetext{
${ }^{*}$ Corresponding author: bartashevichmv@gmail.com
} 
Let us introduce Cartesian coordinate system with axis $O x$ along the flow and axis $O y$ normal to the plate. The velocity profile in the film takes a form

$$
u=3 \bar{u}\left(2 y / h-y^{2} / h^{2}\right) / 2,
$$

where $\bar{u}$ is an average velocity of liquid motion in the film. The process of heat and mass transfer at film absorption is described by the equation of heat conductivity and diffusion:

$$
\begin{aligned}
& u \partial T / \partial x=a \partial^{2} T / \partial y^{2} \\
& u \partial C / \partial x=D \partial^{2} C / \partial y^{2}
\end{aligned}
$$

with the following boundary conditions. At the inlet for $x=0$ :

$$
T=T_{0}, C=C_{0} .
$$

At the interface for $y=h$, similarly to $[1,4]$ there is the equilibrium solution-vapor system:

$$
C_{i}=k_{1}-k_{2} T_{i},-\lambda \partial T / \partial y=-r_{a}\left(\rho D /\left(1-C_{0}\right)\right) \partial C / \partial y \text {. }
$$

This equilibrium condition connects the equilibrium temperature with concentration. On the wall at $y=0$, we set:

$$
\lambda \partial T /\left.\partial y\right|_{y=0}=q_{w}, \partial C /\left.\partial y\right|_{y=0}=0
$$

where $q_{w}$ is heat flux on the wall. The heat transfer coefficient $\alpha_{w}$ is describing the heat transfer from the liquid film to a wall and defined from the relation $q_{w}=\alpha_{w}\left(T_{f}-T_{w}\right)$, where $T_{w}$ is a dimensional wall temperature, $T_{f}=\int_{0}^{1} u T d y / \int_{0}^{1} u d y$ is dimensional bulk temperature of solution. Let us use dimensionless variables $\xi=x /(P e h), \quad \eta=y / h$, $v=u / \bar{u}=3\left(2 \eta-\eta^{2}\right) / 2, \quad \theta=\left(T-T_{0}\right) /\left(T_{e}-T_{0}\right), \quad \gamma=\left(C-C_{0}\right) /\left(C_{e}-C_{0}\right) . \quad$ Here $C_{e}=k_{1}-k_{2} T_{0}$ is equilibrium concentration, corresponding to initial temperature of solution $T_{0}, \quad T_{e}$ is equilibrium temperature, corresponding to initial concentration $C_{0}$ ( $C_{0}=k_{1}-k_{2} T_{e}$ ), similarly to [1]. Pe $=\bar{u} h / a$ is Pecklet number. The system of equations (1) - (2), reduced to the dimensionless form, is written as:

$$
\begin{aligned}
& v \partial \theta / \partial \xi=\partial^{2} \theta / \partial \eta^{2} \\
& v \partial \gamma / \partial \xi=\operatorname{Le} \partial^{2} \gamma / \partial \eta^{2},
\end{aligned}
$$

here $\mathrm{Le}=D / a$. The boundary conditions at the inlet for $\xi=0$ :

$$
\theta=0, \gamma=0
$$

At the interface for $\eta=1$, similarly to [1]:

$$
\theta=\theta_{i}, \gamma=\gamma_{i}
$$




$$
\theta_{i}+\gamma_{i}=1, \operatorname{KaLe} \partial \gamma / \partial \eta=\partial \theta / \partial \eta
$$

Here $\mathrm{Ka}=r_{a}\left(C_{e}-C_{0}\right) /\left(C_{P}\left(T_{e}-T_{0}\right)\left(1-C_{0}\right)\right)$. On the wall at $\eta=0$, we obtain:

$$
\partial \theta /\left.\partial \eta\right|_{\eta=0}=\bar{q}_{w}, \partial \gamma /\left.\partial \eta\right|_{\eta=0}=0
$$

where $\bar{q}_{w}$ is dimensionless heat flux.

\section{Numerical calculations}

Numerical calculations were performed by the finite-difference method. The relationship of temperature and concentration along the interface was calculated via conjugated calculation of the modified coefficients, taking into account the conditions at the interface.

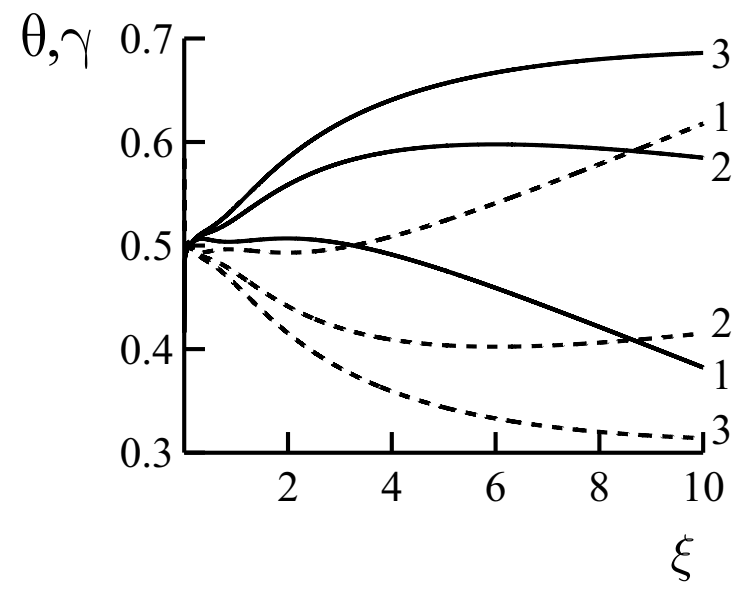

Fig. 1. Dimensionless temperature (solid lines) and concentration (dashed lines) on the film surface. 1 $-\bar{q}_{w}=0.2 ; 2-\bar{q}_{w}=0.1 ; 3-\bar{q}_{w}=0.05$.

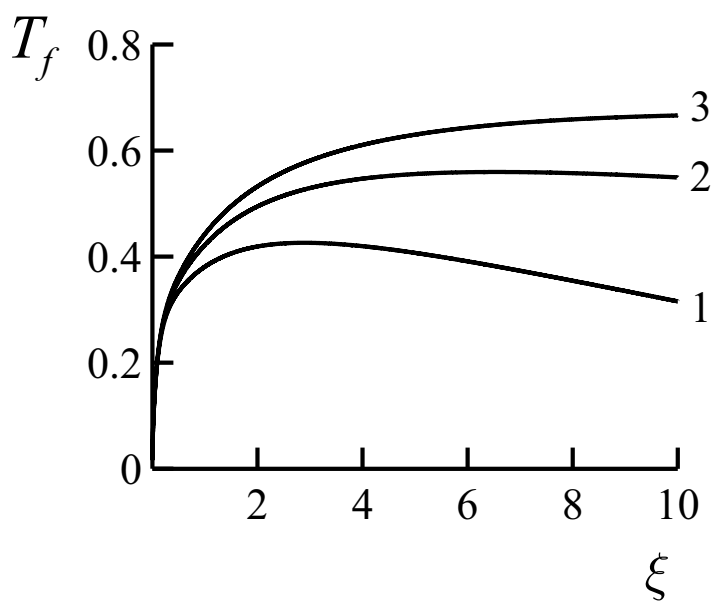

Fig. 2. Dimensionless bulk temperature of solution. $1-\bar{q}_{w}=0.2 ; 2-\bar{q}_{w}=0.1 ; 3-\bar{q}_{w}=0.05$. 
Calculations were carried out at $\mathrm{Ka}=10$, Le $=0.01$. Calculated distributions of dimensionless temperature and concentration along the film surface are shown in Fig. 1 for different heat fluxes on the wall $\bar{q}_{w}$. Dimensionless bulk temperature of solution is shown in Fig.2. Dependences of Nusselt number on longitudinal coordinate are presented in Fig. 3 for different heat fluxes along the initial region of the flow.

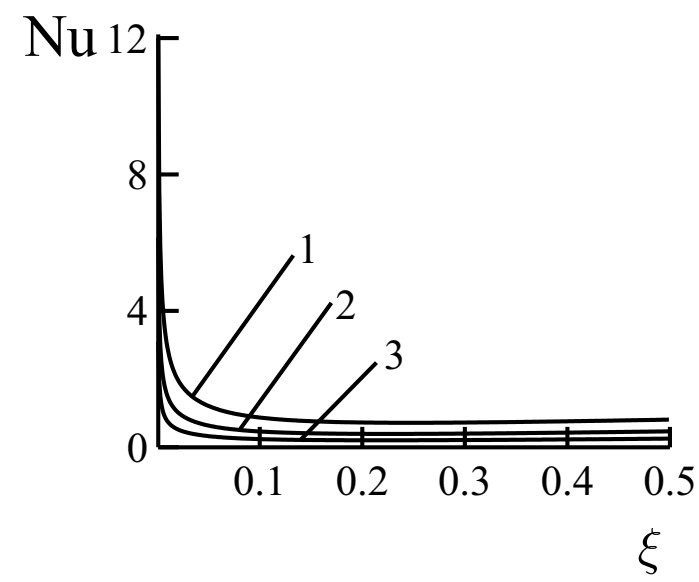

Fig. 3. Nusselt number along the film-flow direction at the initial region. $1-\bar{q}_{w}=0.2 ; 2-\bar{q}_{w}=0.1$; $3-\bar{q}_{w}=0.05$.

\section{Conclusions}

The problem of conjugated heat and mass transfer at film absorption on a cooled wall with a given constant heat flux is studied numerically. The analytical solution is used as an inlet condition for further numerical solution by the finite-difference method. The key dimensionless criteria characterizing heat and mass transfer at absorption on the falling liquid film have been defined.

This work was carried out at the Kutateladze Institute of Thermophysics SB RAS and financially supported by the Russian Science Foundation (project number 15-19-10025).

\section{References}

1. V.E. Nakoryakov, N.I.Grigorieva, Non-Isothermal Absorption in Thermal Transformers (Nauka, Novosibirsk, 2010)

2. S. H.Chiang, H. L. Toor, AlChE J. 10, 398 (1964)

3. N. I.Grigorieva, V. E. Nakoryakov, J. Engin. Phy. Thermophys. 33, 1349 (1977)

4. G.Grossman, International J. Heat Mass Transf. 26, 357 (1983)

5. D. Killion, S. Garimella, Int. J. Refrigeration. 24, 755 (2001)

6. V.E.Nakoryakov, N.I.Grigoryeva, M.V. Bartashevich, Int. J. Heat Mass Transf. 54, 4485 (2011)

7. M. Mittermaier, F. Ziegler, Int. J. Refrigeration. 59, 91 (2015)

8. M. Mortazavi, S. Moghaddam, Int. J. Refrigeration. 66, 93 (2016)

9. E. Garcia-Rivera, J. Castro, J. Farnos, A. Oliva, Int. J. Thermal Sci. 109, 342 (2016) 